\title{
ANALYTICAL SOLUTIONS FOR BENDING, BUCKLING AND VIBRATION ANALYSIS OF FUNCTIONALLY GRADED CYLINDRICAL PANEL
}

\author{
Duong Thanh Huan ${ }^{1,}$, Tran Minh Tu ${ }^{2}$, Tran Huu Quoc ${ }^{2}$ \\ ${ }^{I}$ Vietnam National University of Agriculture, Trau Quy, Gia Lam, Ha Noi, Vietnam \\ ${ }^{2}$ University of Civil Engineering, 55 Giai Phong Road, Hai Ba Trung District, Ha Noi, Vietnam \\ "Email:_duongthanh49@gmail.com
}

Received: 7 November 2016; Accepted for publication: 21 February 2017

\begin{abstract}
The main purpose of this article is to present analytical solutions for bending, buckling and free vibration analysis of cylindrical panel, which are composed of functionally graded materials (FGMs). Equations of motion are derived using Hamilton's principle. The first-order shear deformation theory is used for developing Navier's solutions of simply supported cylindrical panel. Comparison studies are presented to verify the validity of present solution. It is found that the presented results are close to those existing. The effect of volume fraction distributions, panel aspect ratio, and side-to-thickness ratio on the deflections, buckling loads and natural frequencies is also investigated.
\end{abstract}

Keywords: functionally graded cylindrical shell, shear deformation theory, bending, buckling, vibration.

\section{INTRODUCTION}

The concept of FGM was proposed in 1984 by Japanese's scientists as a new class of materials that can resist high temperature. Since then FGMs are being increasingly used in the aeronautical and aerospace industry as well as in other fields of modern technology. FGMs are microscopically inhomogeneous, that exhibit continuous variation of material properties from one surface to another and thus eliminate the stress concentration generally found in conventional laminated composites. The fact shows that having a good understanding of the structural and dynamic behavior such as the deformation characteristic, natural frequencies, critical buckling loads are extremely needed.

Actually, there are a majority of researchers who used both of three-dimensional and twodimensional theories for static and dynamic behavior analysis of cylindrical shells with different boundary conditions and materials. Evidence for this can be found in the following particular studies. First of all, in 1989, a study of bending, vibration and buckling of cross-ply circular cylindrical shells with various shell theories was given by Khdeir, Reddy, and Frederick [1]. 
Next, an elasticity approach was proposed by Varadan and Bhaskar in 1991 [2] for bending analysis of laminated orthotropic cylindrical shell. Then, Soldatos and Ye [3] presented threedimensional static, dynamic, thermoelastic and buckling analysis of homogeneous and laminated composite cylinders in 1994. Some simple solutions for buckling loads of thin and moderately thick laminated composite cylindrical shells and panels were also presented by Geier and Singh in 1997 [4]. Later, in 2007, Matsunaga [5] studied vibration and buckling behaviors of cross-ply laminated composite circular cylindrical shells according to a global higher-order theory. Moreover, Zhao, Lee, and Liew [6] used the element-free kp-Ritz method to analyze the static behavior and free vibration of FGM shells subjected to mechanical or thermomechanical loading in 2009. Another study of Zhao and Liew [10] in 2010 could not be ignored here is that a meshfree method for analysis of the thermal and mechanical buckling of functionally graded cylindrical shell panels. Recently, in 2014, Shadmehri, Hoa, and Hojjati [7] investigated the effect of displacement field on bending, buckling and vibration of cross - ply circular cylindrical shells using the first-order shear deformation theory. In addition, at this time, in 2014, Su, Z., Jin, G., \& Ye, T. [9] used Rayleigh-Ritz method according to the first-order shear deformation shell theory for free vibration analysis of moderately thick functionally graded open shells with general boundary conditions.

On the other hand, there have been a majority of studies including static and dynamic behavior or linear or non-linear analysis about functionally graded cylindrical panel which are subjected to thermal/mechanical loads or in combination of these loads. It takes into account some typical studies and researchers as [11-17].

However, most of these studies related to laminated composite materials and rare literature has been reported for FGM cylindrical shell and panel. Therefore, it is essential to formulate a suitable model which can be used for solving three problems of bending, bucking and free vibration in a combination for FG cylindrical shells.

In this paper, an analytical solutions based on the first shear deformation theory are used for analyzing bending, buckling and vibration of FG cylindrical panel. The numerical results are investigated and discussed with the other author and the different solution.

\section{FORMULATION OF THE PROBLEM AND MATERIAL PROPERTIES}

Consider a functionally graded cylindrical panel with length $L$, radius $R$, span angle $\varphi$ and thickness $h$ (Fig.1). The elastic material properties vary through the panel thickness according to the volume fractions of the constituents. The top surface $(z=h / 2)$ of the panel is ceramic-rich, whereas the bottom surface $(z=-h / 2)$ is metal rich. The effective properties of the functionally graded material at any thickness coordinate $z$ can be expressed following power law distribution as [6]:

$$
P(z)=\left(P_{c}-P_{m}\right) \cdot g(z)+P_{m}
$$

with

$$
g(z)=\left(\frac{1}{2}+\frac{z}{h}\right)^{p}(0 \leq p \leq \infty)
$$

where $P(z)$ denotes the effective material properties, $P_{\mathrm{m}}$ and $P_{\mathrm{c}}$ represent the properties of the metal and ceramic, respectively, $g(z)$ is the volume fraction function and $p$ is the volume fraction exponent. The effective material properties of the cylindrical panel, including Young's modulus E, density $\rho$ vary according to Eq. (1) and Poisson's coefficient is assumed to be constant. 


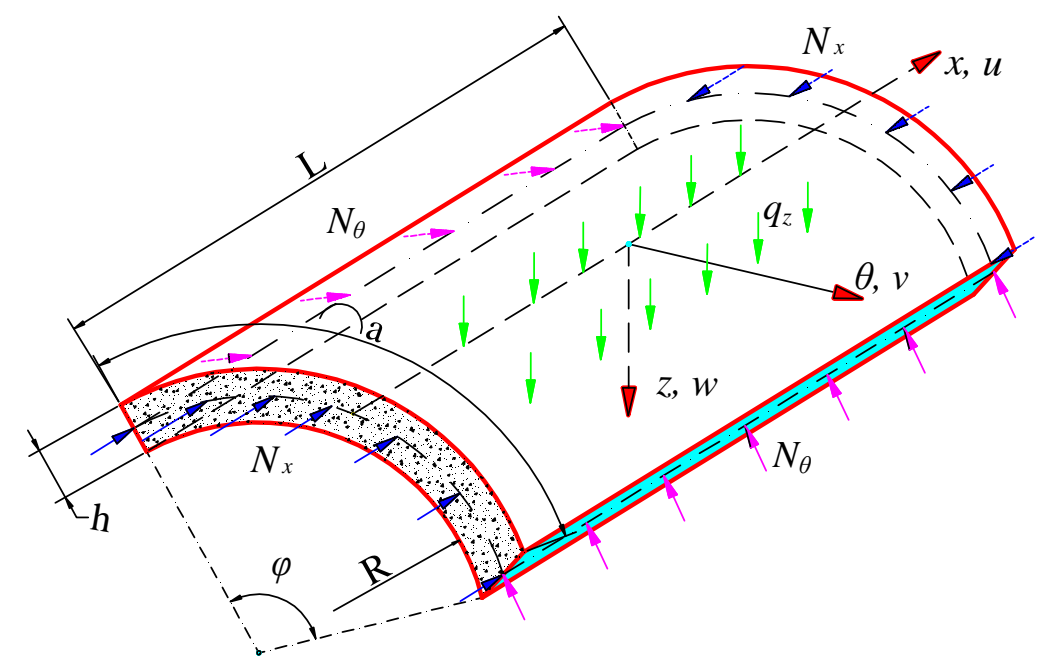

Figure 1. Geometry of the cylindrical panel.

\section{THEORETICAL FORMULATION}

\subsection{Kinematic relations}

The displacement field is assumed according to the first-order shear deformation theory. The three displacement components $u(x, \theta, z), v(x, \theta, z)$ and $w(x \theta, z)$ at any point in the cylindrical panel domain in the $x, \theta$ and $z$ directions are expanded in Taylor's series in terms of the thickness coordinate and expressed as Reddy [8]:

$$
\begin{aligned}
& u(x, \theta, z, t)=u_{0}(x, \theta, t)+z \cdot \theta_{x}(x, \theta, t) \\
& \mathrm{v}(x, \theta, z, t)=v_{0}(x, \theta, t)+z \cdot \theta_{\theta}(x, \theta, t) \\
& w(x, \theta, z, t)=w_{0}(x, \theta, t)
\end{aligned}
$$

The parameters $u_{0}, v_{0}$ are the in-plane tangential displacements and $w_{0}$ is the transverse displacement of a point $(x, \theta)$ on the mid-surface; $\theta_{x}, \theta_{\theta}$ are rotations of a normal to mid-surface about $\theta$ and $x$ axis, respectively. $t$ presents the time.

The linear strain components are obtained from strain - displacement relations for linear elasticity theory.

$$
\{\varepsilon\}=\left\{\varepsilon_{x}, \varepsilon_{\theta}, \gamma_{x \theta}, \gamma_{\theta z}, \gamma_{x z}\right\} \text { or }\{\varepsilon\}=\left\{\varepsilon_{0}\right\}+z\{k\}
$$

where:

$$
\begin{aligned}
& \left\{\varepsilon_{0}\right\}=\left\{\varepsilon_{x 0}, \varepsilon_{y 0}, \gamma_{x \theta 0}, \gamma_{\theta z 0}, \gamma_{x z 0}\right\}=\left\{\frac{\partial u_{o}}{\partial x}, \frac{\partial v_{0}}{\partial \theta}+\frac{w_{0}}{R}, \frac{\partial u_{0}}{\partial \theta}+\frac{\partial v_{0}}{\partial x}, \frac{\partial w_{0}}{\partial \theta}+\theta_{\theta}-\frac{v_{0}}{R}, \frac{\partial w_{0}}{\partial x}+\theta_{x}\right\} \\
& \{k\}=\left\{k_{x}, k_{\theta}, k_{x \theta}, 0,0\right\}=\left\{\frac{\partial \theta_{x}}{\partial x}, \frac{\partial \theta_{\theta}}{\partial_{\theta}}, \frac{\partial \theta_{x}}{\partial \theta}+\frac{\partial \theta_{\theta}}{\partial_{x}}, 0,0\right\}
\end{aligned}
$$




\subsection{Stress - strain relation}

The linear constitutive relation of an FG cylindrical panel can be written as

$$
\left\{\begin{array}{l}
\sigma_{x} \\
\sigma_{\theta} \\
\sigma_{x \theta} \\
\sigma_{x z} \\
\sigma_{\theta z}
\end{array}\right\}=\left[\begin{array}{ccccc}
C_{11} & C_{12} & 0 & 0 & 0 \\
C_{12} & C_{22} & 0 & 0 & 0 \\
0 & 0 & C_{66} & 0 & 0 \\
0 & 0 & 0 & C_{66} & 0 \\
0 & 0 & 0 & 0 & C_{66}
\end{array}\right] \cdot\left\{\begin{array}{l}
\varepsilon_{x} \\
\varepsilon_{\theta} \\
\gamma_{x \theta} \\
\gamma_{x z} \\
\gamma_{\theta z}
\end{array}\right\}=[C] \cdot\left\{\begin{array}{l}
\varepsilon_{x}^{0} \\
\varepsilon_{\theta}^{0} \\
\gamma_{x \theta}^{0} \\
\gamma_{x z}^{0} \\
\gamma_{\theta z}^{0}
\end{array}\right\}+z \cdot[C] \cdot\left\{\begin{array}{l}
k_{x} \\
k_{\theta} \\
k_{x \theta} \\
0 \\
0
\end{array}\right\}
$$

The elements of stiffness matrix are defined as follows:

$$
C_{11}=C_{22}=\frac{E(z)}{1-v^{2}} ; C_{12}=\frac{v \cdot E(z)}{1-v^{2}} ; C_{66}=\frac{E(z)}{2(1+v)}=G ; v \text { - Poison ratio }
$$

\subsection{Equations of motion}

In general way, the equations of motion are derived using the the Hamilton's principle and can be written as Reddy [8].

$$
\begin{array}{ll}
\frac{\partial N_{x}}{\partial x}+\frac{\partial N_{x \theta}}{R \partial \theta}+q_{x}=I_{0} \frac{\partial^{2} u_{0}}{\partial t^{2}}+I_{1} \frac{\partial^{2} \theta_{x}}{\partial t^{2}} ; & \frac{\partial M_{x}}{\partial x}+\frac{\partial M_{x \theta}}{\partial \theta}-Q_{x}=I_{1} \frac{\partial^{2} u_{0}}{\partial t^{2}}+I_{2} \frac{\partial^{2} \beta_{x}}{\partial t^{2}} \\
\frac{\partial N_{x \theta}}{\partial x}+\frac{\partial N_{\theta}}{\partial \theta}+\frac{Q_{\theta}}{R}+q_{\theta}=I_{0} \frac{\partial^{2} v_{0}}{\partial t^{2}}+I_{1} \frac{\partial^{2} \theta_{\theta}}{\partial t^{2}} ; & \frac{\partial M_{\theta}}{\partial \theta}+\frac{\partial M_{x \theta}}{\partial x}-Q_{\theta}=I_{1} \frac{\partial^{2} v_{0}}{\partial t^{2}}+I_{2} \frac{\partial^{2} \beta_{\theta}}{\partial t^{2}} ; \\
\frac{\partial Q_{x}}{\partial x}+\frac{\partial Q_{\theta}}{R \partial \theta}-\frac{N_{\theta}}{R}-N\left(w_{0}\right)+q_{z}=I_{0} \frac{\partial^{2} w_{0}}{\partial t^{2}} ; &
\end{array}
$$

where $N\left(w_{0}\right)=\bar{N}_{x} \cdot \frac{\partial^{2} w_{0}}{\partial x^{2}}+\bar{N}_{\theta} \cdot \frac{\partial^{2} w_{0}}{\partial \theta^{2}}$ are compressive in-plane loads and $\left(q_{x}, q_{\theta}, q_{z}\right)$ are distributed body forces acting on the mid-face. Force resultants, moment resultants and inertias in Eqs. (4) are defined as:

$$
\begin{aligned}
& \left\{\begin{array}{lll}
N_{x} & N_{\theta} & N_{x \theta}
\end{array}\right\}=\int_{-h / 2}^{h / 2}\left\{\begin{array}{lll}
\sigma_{x} & \sigma_{\theta} & \sigma_{x \theta}
\end{array}\right\} d z ; \quad\left\{Q_{x} \quad Q_{\theta}\right\}=K_{s} \int_{-h / 2}^{h / 2}\left\{\begin{array}{ll}
\sigma_{x z} & \sigma_{\theta z}
\end{array}\right\} d z ;
\end{aligned}
$$

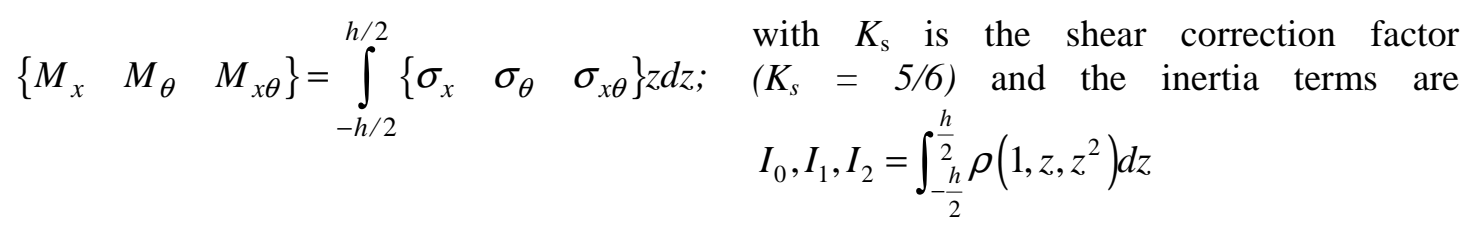

By substituting the stresses from Eqs. (4) into Eqs. (6), the force and moment resultant can be written in terms of displacement variable namely $\left(u, v, w, \theta_{x}, \theta_{\theta}\right)$. Now, if one substitutes these relations into Eqs. (5), the governing equations for the FG cylindrical panel are obtained in terms of displacement variables. 


\section{CLOSED-FORM SOLUTIONS FOR THE SIMPLY SUPPORTED CYLINDRICAL PANEL}

Consider a simply supported cylindrical panel with axial and circumferential lengths are denoted by $L$ and $a$, respectively under only compressive in-plane axial load $\left(\widehat{N}_{x}=-N^{0}, \widehat{N}_{\theta}=0\right)$ and the distributed body force acting on the mid-face in the vertical direction $\left(q_{\mathrm{z}}\right)$. Based on the Navier approach, the displacement unknowns satisfying the simply supported boundary conditions can be expressed in the following forms:

$$
\begin{aligned}
& u_{0}(x, \theta, t)=\sum_{m=1}^{\infty} \sum_{n=1}^{\infty} U_{0 m n} \cos (\alpha x) \sin (\beta \theta) \cdot e^{-i \omega t} ; \quad \theta_{x}(x, \theta, t)=\sum_{m=1}^{\infty} \sum_{n=1}^{\infty} \theta_{x m n} \cos (\alpha x) \sin (\beta \theta) \cdot e^{-i \omega t} \\
& v_{0}(x, \theta, t)=\sum_{m=1}^{\infty} \sum_{n=1}^{\infty} V_{0 m n} \sin (\alpha x) \cos (\beta \theta) \cdot e^{-i \omega t} ; \quad \theta_{\theta}(x, \theta, t)=\sum_{m=1}^{\infty} \sum_{n=1}^{\infty} \theta_{\theta m n} \sin (\alpha x) \cos (\beta \theta) \cdot e^{-i \omega t} \\
& w_{0}(x, \theta, t)=\sum_{m=1}^{\infty} \sum_{n=1}^{\infty} W_{0 m n} \sin (\alpha x) \sin (\beta \theta) \cdot e^{-i \omega t} ;
\end{aligned}
$$

where $i=\sqrt{-1}, \alpha=m \pi / L, \beta=n \pi / \varphi, m, n=1,3,5, \ldots ; \omega$ is the natural frequency. The transverse load $q$ is expanded in the double-Fourier sine series as

$$
q_{z}(x, \theta)=\sum_{m=1}^{\infty} \sum_{n=1}^{\infty} Q_{m n} \sin (\alpha x) \sin (\beta \theta)
$$

where

$$
Q_{m n}=\frac{4}{L a} \int_{0}^{L} \int_{0}^{a} q_{z}(x, \theta) \sin (\alpha x) \sin (\beta \theta) d x d \theta= \begin{cases}q_{0} & \text { for sinusoidally distributed load; } \\ \frac{16 q_{0}}{m n \pi^{2}} & \text { for uniformly distributed load; }\end{cases}
$$

By substituting Eq. (7) into Eqs. (5) which are expressed in terms of displacements $(u, v, w$, $\left.\theta_{x}, \theta_{\theta}\right)$ we get the governing equations in the short form in terms of displacement variable namely $\left(U_{0 m n} ; \mathrm{V}_{0 m n} ; \mathrm{W}_{0 m n} ; \theta_{x m n} ; \theta_{\theta m n}\right)$ as follows

$$
\left([S]_{5 \times 5}-\omega^{2}[M]_{5 \times 5}\right) \cdot\left\{X_{m n}\right\}=\{q\}
$$

in which, $[\mathrm{S}]$ and $[\mathrm{M}]$ are elastic stiffness and mass matrices respectively, $\left\{X_{m n}\right\}$ is the displacement field vector, $\{q\}$ is load vector and $\omega$ is the circular natural frequency corresponding with $m$-th mode in $x$-direction and $n$-th mode in $\theta$-direction.

where,

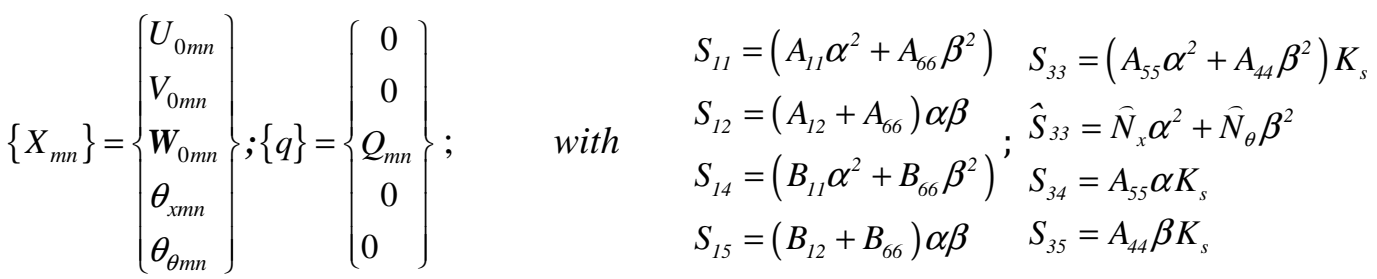




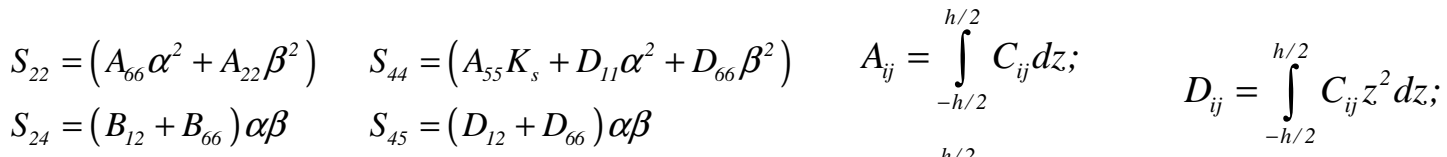

$$
\begin{aligned}
& S_{25}=\left(B_{66} \alpha^{2}+B_{22} \beta^{2}\right) \quad S_{55}=\left(A_{44} K_{s}+D_{66} \alpha^{2}+D_{22} \beta^{2}\right) \quad B_{i j}=\int_{-h / 2}^{h / 2} C_{i j} z d z ; \quad \text { with }(i, j)=(1,2,4,5,6)
\end{aligned}
$$

From the Eq. (8), we have three cases of the problem as follows:

- For bending analysis, the closed-form solution can be obtained by setting the natural frequency $\omega$ and buckling load $N^{0}$ in Eq. (8) equal to zero. Then, the displacements are obtained from the following equation:

$$
[S] .\left\{X_{m n}\right\}=\{q\}
$$

By solving the above equation $(8 \mathrm{a})$, the deflection value $\left(W_{0 m n}\right)$ which is contained in the displacement field vector $\left\{X_{m n}\right\}$, can be obtained.

- For buckling analysis, the closed-form solution can be obtained by setting the frequency $\omega$ and transverse load $q$ in Eq. (8) equal to zero. Thus, the buckling load $N^{0}$ can be obtained from the following equation:

$$
[S] \cdot\left\{X_{m n}\right\}=0
$$

For nontrivial solution, the determinant of the coefficient matrix in Eq. (8b) must be zero. This gives the expression for buckling load $N^{0}$. With each choice of $m$ and $n$, there is a corresponsive unique value of $N^{0}$. The critical buckling load $\left(N_{c r}\right)$ is the smallest value of $N^{0}$.

$$
N_{c r}=\min \left\{N_{(m, n)}^{0}\right\}
$$

- For free vibration analysis, the closed-form solution is obtained by setting the buckling load $N^{0}$ and transverse $q$ in Eq. (8) equal to zero as

$$
\left([S]-\omega^{2}[M]\right) \cdot\left\{X_{m n}\right\}=\{0\}
$$

The resulting equation takes the form of an eigenvalue problem. Thus, the closed-form solution of natural frequency $\omega$ can be obtained from the following equation:

$$
[S]-\omega^{2}[M]=\{0\}
$$

\section{NUMERICAL RESULTS AND DISCUSSION}

For a numerical investigation, the program of matlab codes are developed based on the present theoretical model to analyze bending, buckling and free vibration response of simply supported FG cylindrical panel. The material properties of ceramic and metal are as follows

- Ceramic- $\mathrm{ZrO}_{2}: \mathrm{E}_{\mathrm{c}}=151 \mathrm{GPa} ; \rho_{\mathrm{c}}=3000 \mathrm{~kg} / \mathrm{m}^{3} ; v=0.3$

- $\operatorname{Metal}(\mathrm{Al}): \mathrm{E}_{\mathrm{m}}=70 \mathrm{GPa} ; \rho_{\mathrm{m}}=2707 \mathrm{~kg} / \mathrm{m}^{3} ; v=0.3$

\subsection{Bending analysis}

A fully simply supported FG cylindrical panel subjected to a uniform load is first studied. The geometric properties of the panel are denoted by $L=0.2 \mathrm{~m}, R=1 \mathrm{~m}$ and $\varphi=0.2 \mathrm{rad}$. The 
magnitude of the uniform load is $q_{0}=10^{6} \mathrm{~N} / \mathrm{m}^{2}$. Table 1 shows a validated comparison the displacements in radial direction at the panel center obtained between the proposed method with those from Zhao et al [6] using the element - free $k p$-Ritz method. It could be taken into consideration that those results have a good agreement. Evidence for this can be seen that these differences is not significant (less than $0.40 \%$ ).

Table 1. Nondimensional deflection $\bar{w}\left(\bar{w}=w_{\max } / h\right)$ of cylindrical panel with various volume fraction exponent $p$ and radius to thickness ratio $R / h$.

\begin{tabular}{|c|c|c|c|c|}
\hline \multirow{2}{*}{$R / h$} & \multirow{2}{*}{ Model } & \multicolumn{3}{|c|}{ the volume fraction exponent $p$} \\
\hline & & $p=0.5$ & $p=1$ & $p=2$ \\
\hline \multirow{4}{*}{50} & Zhao. X et al [6] & 0.003824 & 0.004279 & 0.004683 \\
\hline & [Present] & $\mathbf{0 . 0 0 3 8 3 7}$ & 0.004296 & 0.004702 \\
\hline & Difference (\%) & 0.34 & 0.40 & 0.40 \\
\hline & Zhao. X et al [6] & 0.05425 & 0.06072 & 0.06658 \\
\hline \multirow[t]{2}{*}{100} & [Present] & 0.05441 & 0.06091 & 0.06679 \\
\hline & Difference (\%) & 0.29 & 0.31 & 0.31 \\
\hline \multirow{3}{*}{200} & Zhao. X et al [6] & 0.6503 & 0.7283 & 0.8057 \\
\hline & [Present] & 0.6501 & 0.7281 & 0.8056 \\
\hline & Difference (\%) & 0.03 & 0.03 & 0.01 \\
\hline
\end{tabular}

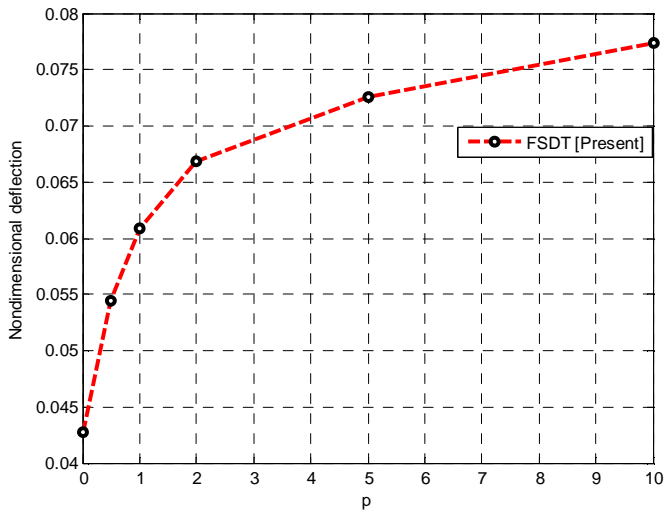

Figure 2. Nondimensional deflection $\bar{w}$ versus the volume fraction exponent $p$.

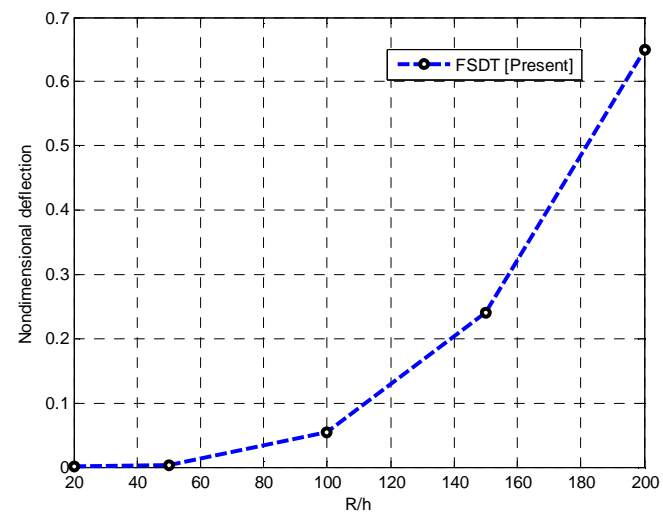

Figure 3. Nondimensional deflection $\bar{w}$ versus radius to thickness ratio $R / h$.

Figures 2 and 3 show the effect of the volume fraction exponent $p$ and radius-to-thickness ratio on dimensionless center deflection of cylindrical panel. It can be seen clearly that, as a general trend, the dimensionless deflection increases dramatically for both case of the volume fraction exponent $p$ and radius-to-thickness ratio have increasing.

\subsection{Vibration analysis}

The natural frequencies of FG cylindrical panel is investigated in this section. A cylindrical FG panel with $\varphi=0.1 \mathrm{rad}$, thickness to length ratio $h / L=0.1$ is considered. The 
nondimensional frequencies with various volume fraction exponent $(p)$ and radius to length ratio $(R / L)$ are given in Table 2 . The obtained results are compared with those given by Su, Jin, and Ye [9] using Rayleigh-Ritz method. It is seen clearly that the results are generally in very good agreement.

Table 2. Nondimensional frequencies $\bar{\omega}\left(\bar{\omega}=\left(\omega \cdot L^{2} \cdot \sqrt{\rho_{C} / E_{C}}\right) / h\right)$ of cylindrical panel with the different volume fraction exponent $p$ and radius to length ratio $R / L$.

\begin{tabular}{|c|c|c|c|c|c|c|c|}
\hline \multirow{2}{*}{$R / L$} & \multirow{2}{*}{ Model } & \multicolumn{6}{|c|}{ the volume fraction exponent $p$} \\
\hline & & $p=0$ & $p=0.5$ & $p=1$ & $p=2$ & $p=5$ & $p=10$ \\
\hline \multirow{3}{*}{2} & Su. $\mathrm{Z}$ et al [9] & 6.1674 & 5.5622 & 5.2964 & 5.0895 & 4.9117 & 4.7787 \\
\hline & [Present] & 6.1755 & 5.5693 & 5.3036 & 5.0969 & 4.9203 & 4.7877 \\
\hline & Difference (\%) & 0.13 & 0.13 & 0.14 & 0.15 & 0.17 & 0.19 \\
\hline \multirow{3}{*}{5} & Su. $Z$ et al [9] & 5.8364 & 5.2532 & 5.0052 & 4.8241 & 4.6755 & 4.5506 \\
\hline & [Present] & 5.8580 & 5.2710 & 5.0225 & 4.8414 & 4.6939 & 4.5692 \\
\hline & Difference $(\%)$ & 0.37 & 0.34 & 0.34 & 0.36 & 0.39 & 0.41 \\
\hline \multirow{3}{*}{20} & Su. Z et al [9] & 5.7742 & 5.1971 & 4.9533 & 4.7774 & 4.6338 & 4.5096 \\
\hline & [Present] & 5.7984 & 5.2174 & 4.9730 & 4.7972 & 4.6546 & 4.5302 \\
\hline & Difference (\%) & 0.42 & 0.39 & 0.40 & 0.41 & 0.45 & 0.45 \\
\hline \multirow{3}{*}{100} & Su. Z et al [9] & 5.7702 & 5.1942 & 4.9509 & 4.7754 & 4.6319 & 4.5075 \\
\hline & [Present] & 5.7946 & 5.2146 & 4.9708 & 4.7955 & 4.6529 & 4.5283 \\
\hline & Difference (\%) & 0.42 & 0.39 & 0.40 & 0.42 & 0.45 & 0.46 \\
\hline
\end{tabular}

Figure 4 and 5 describe the nondimentional frequencies $\bar{\omega}$ of the simply-supported cylindrical FG panel with various volume fraction exponent $(p)$ and radius-to-thickness $(R / h)$ ratio. It is found that the nondimensional frequencies decrease dramatically for both case of the volume fraction exponent $(p)$ and radius-to-thickness ratio $(R / h)$ increasing.

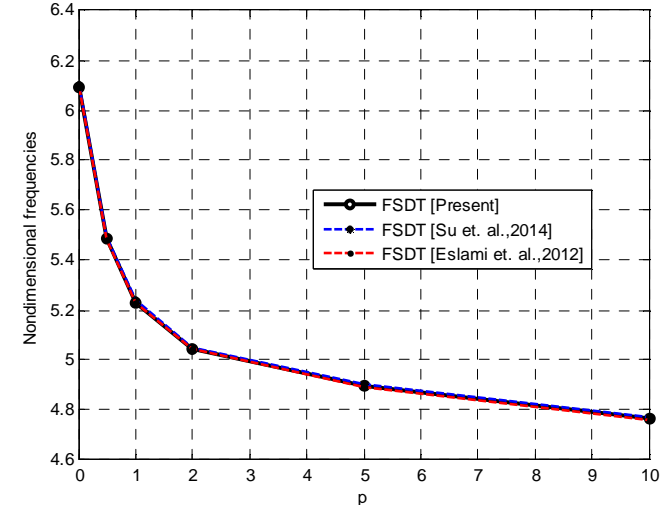

Figure 4. Nondimensional frequencies $\bar{\omega}$ versus the volume fraction exponent $p(h / L=0.02, R / L=20)$.

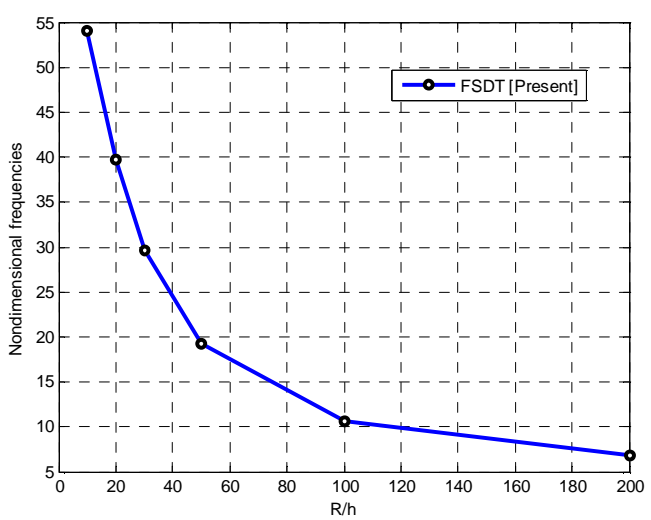

Figure 5. Nondimensional frequencies $\bar{\omega}$ versus radius to thickness ratio $R / h$. 


\subsection{Buckling analysis}

The nondimensional buckling loads $\bar{N}_{c r}$ of FG cylindrical panel with various volume fraction exponent $p$ are tabulated in Table 3. The obtained results are compared with those reported by Zhao and Liew [10] using the mesh-free method. The biggest difference of result is only $2.35 \%$ for case of isotropic material $(p=0)$.

Table 3. Nondimensional buckling load $\bar{N}_{c r}\left(\bar{N}_{c r}=N_{c r} \cdot R /\left(E_{m} \cdot h^{2}\right)\right)$ with the differentvolume fraction exponent $p$.

\begin{tabular}{cccccccc}
\hline \multirow{2}{*}{ Model } & \multicolumn{7}{c}{$\bar{N}_{c r}$} \\
\cline { 2 - 7 } & $p=0$ & $p=0.5$ & $p=1$ & $p=2$ & $p=5$ & $p=10$ & $p=20$ \\
\hline Zhao. X et al [10] & 1.2768 & 1.0390 & 0.9313 & 0.8366 & 0.7464 & 0.6933 & 0.6525 \\
[Present] & $\mathbf{1 . 3 0 7 5}$ & $\mathbf{1 . 0 5 0 0}$ & $\mathbf{0 . 9 3 6 9}$ & $\mathbf{0 . 8 3 9 0}$ & $\mathbf{0 . 7 4 8 4}$ & $\mathbf{0 . 6 9 8 2}$ & $\mathbf{0 . 6 6 0 8}$ \\
Difference (\%) & 2.35 & 1.05 & 0.60 & 0.29 & 0.27 & 0.70 & 1.26 \\
\hline
\end{tabular}

Figures 6 and 7 depict the significant effect of the different values of the volume fraction index $(p)$ and radius to thickness ratio $(R / h)$ on nondimensional buckling load $\bar{N}_{c r}$. According to this consideration, the down trend of nondimensional buckling load is in generally common when the volume fraction index and radius to thickness ratio have an increasing.

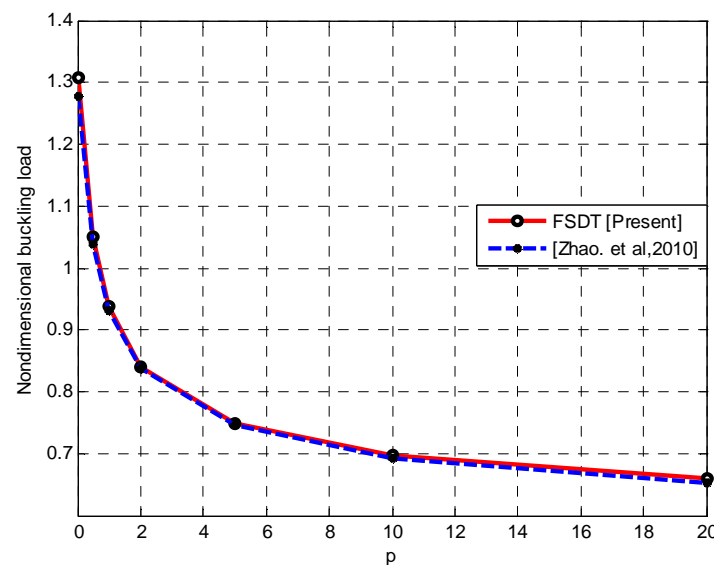

Figure 6. Nondimensional buckling load $\bar{N}_{c r}$ versus Figure 7. Nondimensional buckling load $\bar{N}_{c r}$ versus the volume fraction exponent $p(L=10$ in.; $R=50$ in.; $\theta=0.2 \mathrm{rad})$.

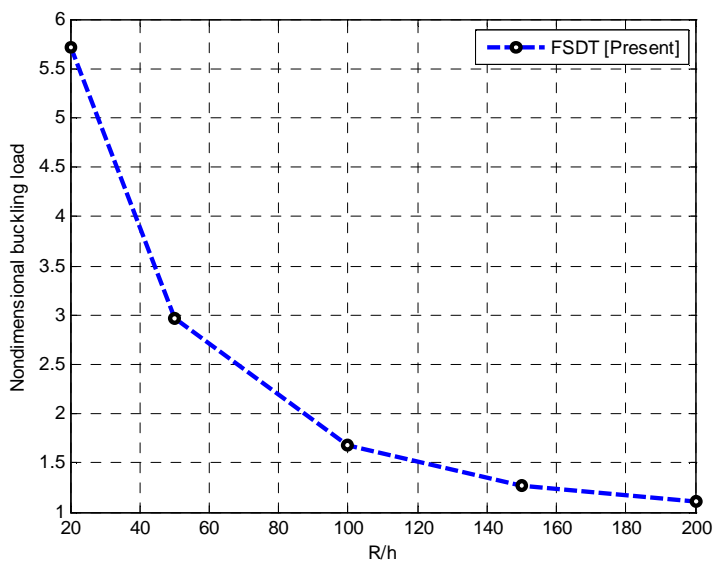

radius to thickness ratio $R / h(p=0.5 ; L=0.2 \mathrm{~m}$; $R=1 \mathrm{~m} ; \theta=0.2 \mathrm{rad})$.

The first four buckling modes of the panel with $R=50$ in. are depicted in Figure 8 which reveal that panel curvature has a significant influence on the buckling mode. 


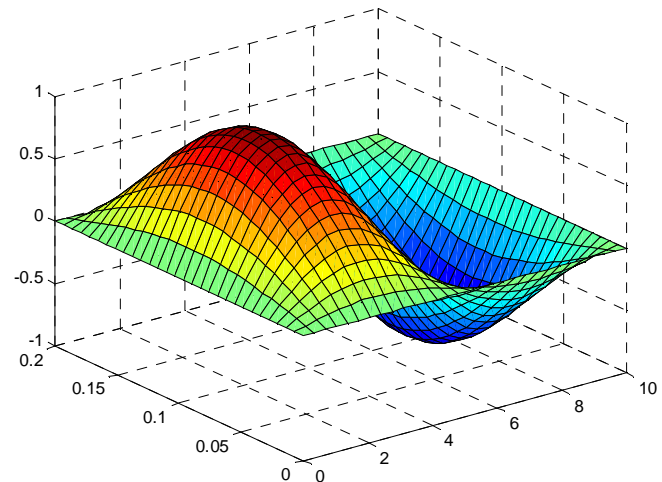

$(m=2 ; n=1)$

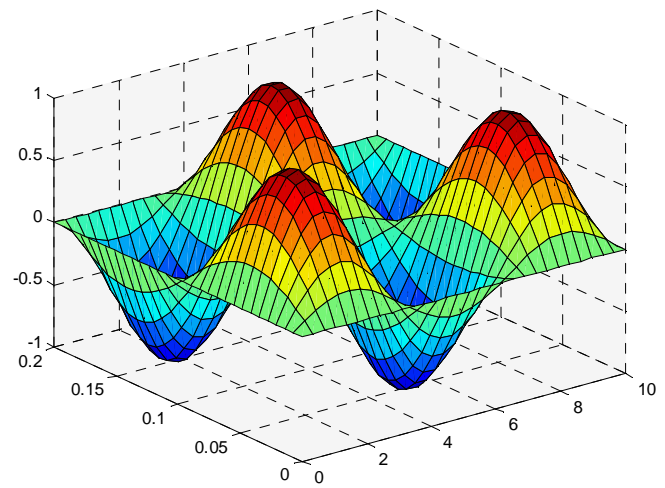

$(m=3 ; n=2)$

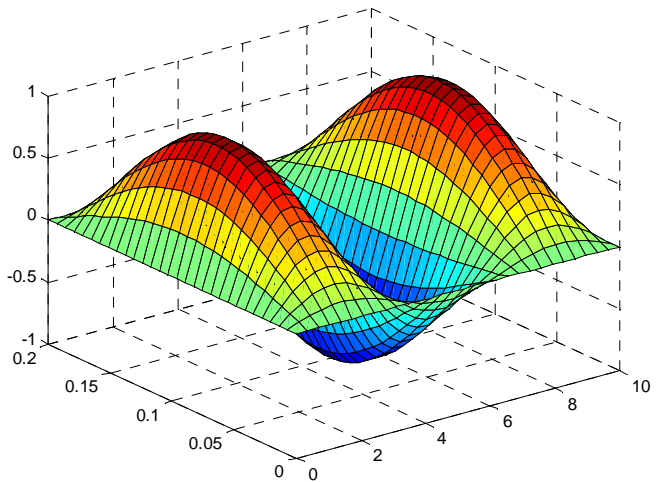

$(m=3 ; n=1)$

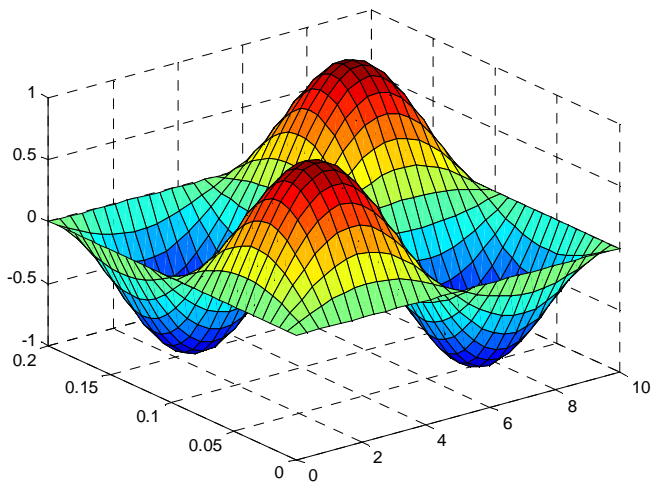

$(m=2 ; n=2)$

Figure 8 . The first four buckling mode shapes.

\section{CONCLUSION}

This paper deals with the analytical solutions for bending, buckling and free vibration analysis of simply supported FG cylindrical panel. Based on the first-order shear deformation theory, the equations of motion are derived from Hamilton's principle.

A series of comparisons and parametric studies have been performed to verify the validity of the analytical solutions and to examine various parameters. Hope that the presented analytical solutions could be useful references for the future researches.

\section{REFERENCES}

1. Khdeir, A., Reddy, J., \& Frederick, D. - A study of bending, vibration and buckling of cross-ply circular cylindrical shells with various shell theories. International Journal of Engineering Science 27 (11) (1989) 1337-1351.

2. Varadan, T., and Bhaskar, K. - Bending of laminated orthotropic cylindrical shells-an elasticity approach. Composite Structures 17 (2) (1991) 141-156. 
3. Soldatos, K. P., \& Ye, J.-Q. - Three-dimensional static, dynamic, thermoelastic and buckling analysis of homogeneous and laminated composite cylinders. Composite Structures 29 (2) (1994) 131-143.

4. Geier, B., \& Singh, G. - Some simple solutions for buckling loads of thin and moderately thick cylindrical shells and panels made of laminated composite material. Aerospace science and technology 1 (1) (1997) 47-63.

5. Matsunaga, H. - Vibration and buckling of cross-ply laminated composite circular cylindrical shells according to a global higher-order theory. International Journal of Mechanical Sciences 49 (9) (2007) 1060-1075.

6. Zhao, X., Lee, Y., and Liew, K. M. - Thermoelastic and vibration analysis of functionally graded cylindrical shells. International Journal of Mechanical Sciences 51(9) (2009) 694707.

7. Shadmehri, F., Hoa, S., and Hojjati, M. - The effect of displacement field on bending, buckling, and vibration of cross-ply circular cylindrical shells. Mechanics of Advanced Materials and Structures 21 (1) (2014) 14-22.

8. Reddy, J. N. - Theory and analysis of elastic plates and shells: CRC press, (2006).

9. Su, Z., Jin, G., and Ye, T. - Free vibration analysis of moderately thick functionally graded open shells with general boundary conditions. Composite Structures 117 (2014) 169-186.

10. Zhao, X., and Liew, K. M. - A mesh-free method for analysis of the thermal and mechanical buckling of functionally graded cylindrical shell panels. Computational Mechanics 45 (4) (2010) 297-310.

11. Duc, N. D., \& Van Tung, H. - Nonlinear analysis of stability for functionally graded cylindrical panels under axial compression. Computational Materials Science 49 (4) (2010) S313-S316.

12. Duc, N. D., and Van Tung, H. - Nonlinear response of pressure-loaded functionally graded cylindrical panels with temperature effects. Composite Structures 92 (7) (2010) 1664-1672.

13. Bich, D. H., Van Dung, D., \& Nam, V. H. - Nonlinear dynamical analysis of eccentrically stiffened functionally graded cylindrical panels.Composite Structures 94 (8) (2012) 24652473.

14. Shao, Z. S., and Wang, T. J. - Three-dimensional solutions for the stress fields in functionally graded cylindrical panel with finite length and subjected to thermal/mechanical loads. International Journal of Solids and Structures 43(13) (2006) 3856-3874.

15. Ootao, Y., \& Tanigawa, Y. - Two-dimensional thermoelastic analysis of a functionally graded cylindrical panel due to nonuniform heat supply.Mechanics Research Communications 32 (4) (2005) 429-443.

16. Shen, H. S. - Postbuckling analysis of axially loaded functionally graded cylindrical panels in thermal environments. International journal of solids and structures 39 (24) (2002) 5991-6010.

17. Yang, J., and Shen, H. S.. - Free vibration and parametric resonance of shear deformable functionally graded cylindrical panels. Journal of Sound and Vibration 261 (5) (2003) 871-893. 